\title{
Uncountably many solutions of first-order neutral nonlinear differential equations
}

\author{
Božena Dorociaková ${ }^{*}$, Martina Kubjatková ${ }^{2}$ and Rudolf Olach²
}

\author{
"Correspondence: \\ bozena.dorociakova@fstroj.uniza.sk \\ 'Department of Applied \\ Mathematics, University of Žilina, \\ Žilina, Slovak Republic \\ Full list of author information is \\ available at the end of the article
}

\begin{abstract}
The article deals with the existence of uncountably many positive solutions which are bounded below and above by positive functions for the first-order nonlinear neutral differential equations. Some examples are included to illustrate the results presented in this article.
\end{abstract}

MSC: Primary 34K40; secondary 34K12

Keywords: neutral differential equation; nonlinear; existence; uncountably many positive solutions; Banach space

\section{Introduction}

In recent years, the study of existence and qualitative properties of solutions for various kinds of neutral delay differential equations has attracted much attention. For related results, we refer the reader to [1-13] and the references cited therein. The authors only considered the existence of solutions which are bounded by positive constants, e.g., in [8, 9, 11-13]. For example, Erbe et al. [6] established a few oscillation and nonoscillation criteria for linear neutral delay differential equation

$$
[x(t)-p(t) x(t-\tau)]^{\prime}+q(t) x(t-\sigma(t))=0, \quad t \geq t_{0} .
$$

Diblík and co-autors in [1-4] studied the existence of positive and oscillatory solutions of differential equations with delay and nonlinear systems in view of Ważievski's retract principle and later extended to retarded functional differential equations by Rybakowski. Zhou [12] deduced the existence of nonoscillatory solutions of the second-order nonlinear neutral differential equations and Lin et al. [9] discussed the existence of nonoscillatory solutions for a third-order nonlinear neutral delay differential equation, and by utilizing Krasnoselskii's fixed point theorem and Schauder's fixed point theorem, they developed some sufficient conditions for the existence of uncountably many nonoscillatory solutions bounded by positive constants. Some interesting results about the existence of nonoscillatory solutions of delay differential equations can also be found in $[1,5]$.

In this paper, we investigate the following nonlinear neutral differential delay differential equations:

$$
\frac{d}{d t}[x(t)-a(t) x(t-\tau)]=p(t) f(x(t-\sigma)), \quad t \geq t_{0}
$$

C 2013 Dorociaková et al.; licensee Springer. This is an Open Access article distributed under the terms of the Creative Commons Attribution License (http://creativecommons.org/licenses/by/2.0), which permits unrestricted use, distribution, and reproduction in any medium, provided the original work is properly cited. 
where $\tau>0, \sigma \geq 0, a \in C\left(\left[t_{0}, \infty\right),(0, \infty)\right), p \in C(R,(0, \infty)), f \in C(R, R), f$ is a nondecreasing function for $x>0$ and $f(x)>0, x>0$.

By a solution of Eq. (1), we mean a function $x \in C\left(\left[t_{1}-\tau, \infty\right), R\right)$ for some $t_{1} \geq t_{0}$ such that $x(t)-a(t) x(t-\tau)$ is continuously differentiable on $\left[t_{1}, \infty\right)$ and such that Eq. (1) is satisfied for $t \geq t_{1}$.

As much as we know, in the literature there is no result for the existence of uncountably many solutions which are bounded below and above by positive functions. This problem is discussed and treated in this paper.

The following fixed point theorem will be used to prove the main results in the next section.

Lemma 1.1 $([6,12]$ Krasnoselskii's fixed point theorem) Let $X$ be a Banach space, let $\Omega$ be a bounded closed convex subset of $X$ and let $S_{1}, S_{2}$ be maps of $\Omega$ into $X$ such that $S_{1} x+$ $S_{2} y \in \Omega$ for every pair $x, y \in \Omega$. If $S_{1}$ is contractive and $S_{2}$ is completely continuous, then the equation

$$
S_{1} x+S_{2} x=x
$$

has a solution in $\Omega$.

\section{The existence of positive solutions}

In this section we consider the existence of uncountably many positive solutions for Eq. (1) which are bounded by two positive functions. We use the notation $m=\max \{\tau, \sigma\}$.

Theorem 2.1 Suppose that there exist bounded from below and from above by the functions $u$ and $v \in C^{1}\left(\left[t_{0}, \infty\right),(0, \infty)\right)$ constants $c>0, K_{2}>K_{1} \geq 0$ and $t_{1} \geq t_{0}+m$ such that

$$
\begin{aligned}
& u(t) \leq v(t), \quad t \geq t_{0}, \\
& v(t)-v\left(t_{1}\right)-u(t)+u\left(t_{1}\right) \geq 0, \quad t_{0} \leq t \leq t_{1}, \\
& \frac{1}{u(t-\tau)}\left(u(t)-K_{1}+\int_{t}^{\infty} p(s) f(v(s-\sigma)) d s\right) \\
& \quad \leq a(t) \leq \frac{1}{v(t-\tau)}\left(v(t)-K_{2}+\int_{t}^{\infty} p(s) f(u(s-\sigma)) d s\right) \leq c<1, \quad t \geq t_{1} .
\end{aligned}
$$

Then Eq. (1) has uncountably many positive solutions which are bounded by the functions $u, v$.

Proof Let $C\left(\left[t_{0}, \infty\right), R\right)$ be the set of all continuous bounded functions with the norm $\|x\|=\sup _{t \geq t_{0}}|x(t)|$. Then $C\left(\left[t_{0}, \infty\right), R\right)$ is a Banach space. We define a closed, bounded and convex subset $\Omega$ of $C\left(\left[t_{0}, \infty\right), R\right)$ as follows:

$$
\Omega=\left\{x=x(t) \in C\left(\left[t_{0}, \infty\right), R\right): u(t) \leq x(t) \leq v(t), t \geq t_{0}\right\} .
$$

For $K \in\left[K_{1}, K_{2}\right]$ we define two maps $S_{1}$ and $S_{2}: \Omega \rightarrow C\left(\left[t_{0}, \infty\right), R\right)$ as follows:

$$
\left(S_{1} x\right)(t)= \begin{cases}K+a(t) x(t-\tau), & t \geq t_{1}, \\ \left(S_{1} x\right)\left(t_{1}\right), & t_{0} \leq t \leq t_{1},\end{cases}
$$




$$
\left(S_{2} x\right)(t)= \begin{cases}-\int_{t}^{\infty} p(s) f(x(s-\sigma)) d s, & t \geq t_{1}, \\ \left(S_{2} x\right)\left(t_{1}\right)+v(t)-v\left(t_{1}\right), & t_{0} \leq t \leq t_{1}\end{cases}
$$

We will show that for any $x, y \in \Omega$, we have $S_{1} x+S_{2} y \in \Omega$. For every $x, y \in \Omega$ and $t \geq t_{1}$ with regard to (4), we obtain

$$
\begin{aligned}
\left(S_{1} x\right)(t)+\left(S_{2} y\right)(t) & =K+a(t) x(t-\tau)-\int_{t}^{\infty} p(s) f(y(s-\sigma)) d s \\
& \leq K+a(t) v(t-\tau)-\int_{t}^{\infty} p(s) f(u(s-\sigma)) d s \\
& \leq K+v(t)-K_{2} \leq v(t) .
\end{aligned}
$$

For $t \in\left[t_{0}, t_{1}\right]$ we have

$$
\begin{aligned}
\left(S_{1} x\right)(t)+\left(S_{2} y\right)(t) & =\left(S_{1} x\right)\left(t_{1}\right)+\left(S_{2} y\right)\left(t_{1}\right)+v(t)-v\left(t_{1}\right) \\
& \leq v\left(t_{1}\right)+v(t)-v\left(t_{1}\right)=v(t) .
\end{aligned}
$$

Furthermore, for $t \geq t_{1}$ we get

$$
\begin{aligned}
\left(S_{1} x\right)(t)+\left(S_{2} y\right)(t) & \geq K+a(t) u(t-\tau)-\int_{t}^{\infty} p(s) f(v(s-\sigma)) d s \\
& \geq K+u(t)-K_{1} \geq u(t) .
\end{aligned}
$$

Let $t \in\left[t_{0}, t_{1}\right]$. With regard to (3), we get

$$
v(t)-v\left(t_{1}\right)+u\left(t_{1}\right) \geq u(t), \quad t_{0} \leq t \leq t_{1} .
$$

Then, for $t \in\left[t_{0}, t_{1}\right]$ and any $x, y \in \Omega$, we obtain

$$
\begin{aligned}
\left(S_{1} x\right)(t)+\left(S_{2} y\right)(t) & =\left(S_{1} x\right)\left(t_{1}\right)+\left(S_{2} y\right)\left(t_{1}\right)+v(t)-v\left(t_{1}\right) \\
& \geq u\left(t_{1}\right)+v(t)-v\left(t_{1}\right) \geq u(t) .
\end{aligned}
$$

Thus we have proved that $S_{1} x+S_{2} y \in \Omega$ for any $x, y \in \Omega$.

We will show that $S_{1}$ is a contraction mapping on $\Omega$. For $x, y \in \Omega$ and $t \geq t_{1}$, we have

$$
\left|\left(S_{1} x\right)(t)-\left(S_{1} y\right)(t)\right|=|a(t)||x(t-\tau)-y(t-\tau)| \leq c\|x-y\| .
$$

This implies that

$$
\left\|S_{1} x-S_{1} y\right\| \leq c\|x-y\| .
$$

Also, for $t \in\left[t_{0}, t_{1}\right]$ the inequality above is valid. We conclude that $S_{1}$ is a contraction mapping on $\Omega$.

We now show that $S_{2}$ is completely continuous. First, we show that $S_{2}$ is continuous. Let $x_{k}=x_{k}(t) \in \Omega$ be such that $x_{k}(t) \rightarrow x(t)$ as $k \rightarrow \infty$. Because $\Omega$ is closed, $x=x(t) \in \Omega$. For 
$t \geq t_{1}$ we have

$$
\begin{aligned}
\left|\left(S_{2} x_{k}\right)(t)-\left(S_{2} x\right)(t)\right| & \leq\left|\int_{t}^{\infty} p(s)\left[f\left(x_{k}(s-\sigma)\right)-f(x(s-\sigma))\right] d s\right| \\
& \leq \int_{t_{1}}^{\infty} p(s)\left|f\left(x_{k}(s-\sigma)\right)-f(x(s-\sigma))\right| d s
\end{aligned}
$$

According to (7), we get

$$
\int_{t_{1}}^{\infty} p(s) f(v(s-\sigma)) d s<\infty
$$

Since $\left|f\left(x_{k}(s-\sigma)\right)-f(x(s-\sigma))\right| \rightarrow 0$ as $k \rightarrow \infty$, by applying the Lebesgue dominated convergence theorem, we obtain that

$$
\lim _{k \rightarrow \infty}\left\|\left(S_{2} x_{k}\right)(t)-\left(S_{2} x\right)(t)\right\|=0
$$

This means that $S_{2}$ is continuous.

We now show that $S_{2} \Omega$ is relatively compact. It is sufficient to show by the Arzela-Ascoli theorem that the family of functions $\left\{S_{2} x: x \in \Omega\right\}$ is uniformly bounded and equicontinuous on $\left[t_{0}, \infty\right)$. The uniform boundedness follows from the definition of $\Omega$. For the equicontinuity, we only need to show, according to the Levitan result [7], that for any given $\varepsilon>0$, the interval $\left[t_{0}, \infty\right)$ can be decomposed into finite subintervals in such a way that on each subinterval all functions of the family have a change of amplitude less than $\varepsilon$. Then, with regard to condition (8), for $x \in \Omega$ and any $\varepsilon>0$, we take $t^{*} \geq t_{1}$ large enough so that

$$
\int_{t^{*}}^{\infty} p(s) f(x(s-\sigma)) d s<\frac{\varepsilon}{2}
$$

Then, for $x \in \Omega, T_{2}>T_{1} \geq t^{*}$, we have

$$
\begin{aligned}
\left|\left(S_{2} x\right)\left(T_{2}\right)-\left(S_{2} x\right)\left(T_{1}\right)\right| \leq & \int_{T_{2}}^{\infty} p(s) f(x(s-\sigma)) d s \\
& +\int_{T_{1}}^{\infty} p(s) f(x(s-\sigma)) d s<\frac{\varepsilon}{2}+\frac{\varepsilon}{2}=\varepsilon .
\end{aligned}
$$

For $x \in \Omega$ and $t_{1} \leq T_{1}<T_{2} \leq t^{*}$, we get

$$
\begin{aligned}
\left|\left(S_{2} x\right)\left(T_{2}\right)-\left(S_{2} x\right)\left(T_{1}\right)\right| & \leq \int_{T_{1}}^{T_{2}} p(s) f(x(s-\sigma)) d s \\
& \leq \max _{t_{1} \leq s \leq t^{t}}\{p(s) f(x(s-\sigma))\}\left(T_{2}-T_{1}\right) .
\end{aligned}
$$

Thus there exists $\delta_{1}=\frac{\varepsilon}{M}$, where $M=\max _{t_{1} \leq s \leq t^{*}}\{p(s) f(x(s-\sigma))\}$, such that

$$
\left|\left(S_{2} x\right)\left(T_{2}\right)-\left(S_{2} x\right)\left(T_{1}\right)\right|<\varepsilon \quad \text { if } 0<T_{2}-T_{1}<\delta_{1} .
$$


Finally, for any $x \in \Omega, t_{0} \leq T_{1}<T_{2} \leq t_{1}$, there exists a $\delta_{2}>0$ such that

$$
\begin{aligned}
\left|\left(S_{2} x\right)\left(T_{2}\right)-\left(S_{2} x\right)\left(T_{1}\right)\right| & =\left|v\left(T_{1}\right)-v\left(T_{2}\right)\right|=\left|\int_{T_{1}}^{T_{2}} v^{\prime}(s) d s\right| \\
& \leq \max _{t_{0} \leq s \leq t_{1}}\left\{\left|v^{\prime}(s)\right|\right\}\left(T_{2}-T_{1}\right)<\varepsilon \quad \text { if } 0<T_{2}-T_{1}<\delta_{2} .
\end{aligned}
$$

Then $\left\{S_{2} x: x \in \Omega\right\}$ is uniformly bounded and equicontinuous on $\left[t_{0}, \infty\right)$, and hence $S_{2} \Omega$ is a relatively compact subset of $C\left(\left[t_{0}, \infty\right), R\right)$. By Lemma 1.1 there is an $x_{0} \in \Omega$ such that $S_{1} x_{0}+S_{2} x_{0}=x_{0}$. We conclude that $x_{0}(t)$ is a positive solution of (1).

Next we show that Eq. (1) has uncountably many bounded positive solutions in $\Omega$. Let the constant $\bar{K} \in\left[K_{1}, K_{2}\right]$ be such that $\bar{K} \neq K$. We infer similarly that there exist mappings $\bar{S}_{1}, \bar{S}_{2}$ satisfying (5), (6), where $K, S_{1}, S_{2}$ are replaced by $\bar{K}, \bar{S}_{1}, \bar{S}_{2}$, respectively. We assume that $x, y \in \Omega, S_{1} x+S_{2} x=x, \bar{S}_{1} y+\bar{S}_{2} y=y$, which are the bounded positive solutions of Eq. (1), that is,

$$
\begin{aligned}
& x(t)=K+a(t) x(t-\tau)-\int_{t}^{\infty} p(s) f(x(s-\sigma)) d s, \quad t \geq t_{1}, \\
& y(t)=\bar{K}+a(t) y(t-\tau)-\int_{t}^{\infty} p(s) f(y(s-\sigma)) d s, \quad t \geq t_{1} .
\end{aligned}
$$

From condition (8) it follows that there exists a $t_{2}>t_{1}$ satisfying

$$
\int_{t_{2}}^{\infty} p(s)[f(x(s-\sigma))+f(y(s-\sigma))] d s<|K-\bar{K}|
$$

In order to prove that the set of bounded positive solutions of Eq. (1) is uncountable, it is sufficient to verify that $x \neq y$. For $t \geq t_{2}$ we get

$$
\begin{aligned}
\mid x(t) & -y(t) \mid \\
= & \mid K+a(t) x(t-\tau)-\int_{t}^{\infty} p(s) f(x(s-\sigma)) d s \\
& -\bar{K}-a(t) y(t-\tau)+\int_{t}^{\infty} p(s) f(y(s-\sigma)) d s \mid \\
\geq & \mid K-\bar{K}+a(t)[x(t-\tau)-y(t-\tau)] \\
& -\int_{t}^{\infty} p(s)[f(x(s-\sigma))-f(y(s-\sigma))] d s \mid \\
\geq & |K-\bar{K}|-a(t)\|x-y\|-\left|\int_{t}^{\infty} p(s)[f(x(s-\sigma))-f(y(s-\sigma))] d s\right| \\
\geq & |K-\bar{K}|-c\|x-y\|-\int_{t}^{\infty} p(s)[f(x(s-\sigma))+f(y(s-\sigma))] d s .
\end{aligned}
$$

Then we have

$$
(1+c)\|x-y\| \geq|K-\bar{K}|-\int_{t}^{\infty} p(s)[f(x(s-\sigma))+f(y(s-\sigma))] d s, \quad t \geq t_{2} .
$$


According to (9) we get that $x \neq y$. Since the interval $\left[K_{1}, K_{2}\right]$ contains uncountably many constants, then Eq. (1) has uncountably many positive solutions which are bounded by the functions $u(t), v(t)$. This completes the proof.

Corollary 2.1 Suppose that there exist bounded from below and from above by the functions $u$ and $v \in C^{1}\left(\left[t_{0}, \infty\right),(0, \infty)\right)$ constants $c>0, K_{2}>K_{1} \geq 0$ and $t_{1} \geq t_{0}+m$ such that (2), (4) hold and

$$
v^{\prime}(t)-u^{\prime}(t) \leq 0, \quad t_{0} \leq t \leq t_{1} .
$$

Then Eq. (1) has uncountably many positive solutions which are bounded by the functions $u, v$.

Proof We only need to prove that condition (10) implies (3). Let $t \in\left[t_{0}, t_{1}\right]$ and set

$$
H(t)=v(t)-v\left(t_{1}\right)-u(t)+u\left(t_{1}\right) .
$$

Then, with regard to (10), it follows that

$$
H^{\prime}(t)=v^{\prime}(t)-u^{\prime}(t) \leq 0, \quad t_{0} \leq t \leq t_{1} .
$$

Since $H\left(t_{1}\right)=0$ and $H^{\prime}(t) \leq 0$ for $t \in\left[t_{0}, t_{1}\right]$, this implies that

$$
H(t)=v(t)-v\left(t_{1}\right)-u(t)+u\left(t_{1}\right) \geq 0, \quad t_{0} \leq t \leq t_{1} .
$$

Thus all the conditions of Theorem 2.1 are satisfied.

Example 2.1 Consider the nonlinear neutral differential equation

$$
[x(t)-a(t) x(t-2)]^{\prime}=p(t) x^{3}(t-1), \quad t \geq t_{0},
$$

where $p(t)=e^{-t}$. We will show that the conditions of Corollary 2.1 are satisfied. The functions $u(t)=0.5, v(t)=2$ satisfy (2) and also condition (10) for $t \in\left[t_{0}, t_{1}\right]=[0,4]$. For the constants $K_{1}=0.5, K_{2}=1$, condition (4) has the form

$$
16 e^{-t} \leq a(t) \leq \frac{1}{2}+\frac{1}{16} e^{-t}, \quad t \geq t_{1}=4
$$

If the function $a(t)$ satisfies (12), then Eq. (11) has uncountably many positive solutions which are bounded by the functions $u, v$.

Example 2.2 Consider the nonlinear neutral differential equation

$$
[x(t)-a(t) x(t-\tau)]^{\prime}=p(t) x^{2}(t-\sigma), \quad t \geq t_{0},
$$

where $\tau, \sigma \in(0, \infty), p(t)=e^{-3 t}$. We will show that the conditions of Corollary 2.1 are satisfied. The functions $u(t)=e^{-2 t}, v(t)=e^{\tau}+e^{-t}, t \geq 1$, satisfy (2) and since

$$
v^{\prime}(t)-u^{\prime}(t)=e^{-t}\left(2 e^{-t}-1\right)<0 \quad \text { for } t \in[1,2]
$$


condition (10) is also satisfied. For the constants $K_{1}=0, K_{2}=e^{\tau}-1$, condition (4) has the form

$$
e^{-2 \tau}+\frac{1}{3} e^{-t}+\frac{1}{2} e^{-2 t+\sigma-\tau}+\frac{1}{5} e^{-3 t+2(\sigma-\tau)} \leq a(t) \leq e^{-\tau}+\frac{e^{-7 t+4 \sigma-\tau}}{7\left(1+e^{-t}\right)}, \quad t \geq 2 .
$$

For $\tau=\sigma=1$ we get

$$
e^{-2}+\frac{1}{3} e^{-t}+\frac{1}{2} e^{-2 t}+\frac{1}{5} e^{-3 t} \leq a(t) \leq e^{-1}+\frac{e^{-7 t+3}}{7\left(1+e^{-t}\right)}, \quad t \geq t_{1}=2 .
$$

If the function $a(t)$ satisfies (14), then Eq. (13) has uncountably many solutions which are bounded by the functions $u, v$.

Example 2.3 Consider the nonlinear neutral differential equation

$$
[x(t)-a(t) x(t-\tau)]^{\prime}=p(t) x^{3}(t-\sigma), \quad t \geq t_{0},
$$

where $\tau, \sigma \in(0, \infty), p(t)=e^{-t}$. We will show that the conditions of Corollary 2.1 are satisfied. The functions $u(t)=e^{-t}, v(t)=e^{\tau}+2 e^{-t}, t \geq 1$ satisfy (2) and also (10)

$$
v^{\prime}(t)-u^{\prime}(t)=-e^{-t}<0 \quad \text { for } t \in[1,3.2] .
$$

For the constants $K_{1}=1, K_{2}=e^{\tau}-1$, where $\tau>\ln 2, t \geq 3.2$, condition (4) has the form

$$
e^{-\tau}\left(1-e^{t}+e^{3 \tau}+3 e^{2 \tau+\sigma-t}+4 e^{\tau+2 \sigma-2 t}+2 e^{3 \sigma-3 t}\right) \leq a(t) \leq e^{-\tau}+\frac{e^{3 \sigma-\tau-4 t}}{4\left(1+2 e^{-t}\right)}
$$

For $\tau=\sigma=1$ and $t \geq 3.2$, we have

$$
e^{-1}\left(1-e^{t}+e^{3}+3 e^{3-t}+4 e^{3-2 t}+2 e^{3(1-t)}\right)<0 .
$$

Then for $a(t)$, which satisfies the inequalities

$$
0<a(t) \leq e^{-1}+\frac{e^{2(1-2 t)}}{4\left(1+2 e^{-t}\right)}, \quad t \geq t_{1} \geq 3.2,
$$

Eq. (11) has uncountably many solutions which are bounded by the functions $u, v$.

\section{Competing interests}

The authors declare that they have no competing interests.

Authors' contributions

The authors have made the same contribution. All authors read and approved the final manuscript.

\section{Author details}

'Department of Applied Mathematics, University of Žilina, Žilina, Slovak Republic. ${ }^{2}$ Department of Mathematics, University of Žilina, Žilina, Slovak Republic.

\section{Acknowledgements}

The research was supported by the grant 1/0090/09 of the Scientific Grant Agency of the Ministry of Education of the Slovak Republic.

Received: 19 December 2012 Accepted: 29 April 2013 Published: 16 May 2013 


\section{References}

1. Boichuk, A, Diblík, J, Khusainov, D, Rưžičková, M: Fredholm's boundary-value problems for differential systems with a single delay. Nonlinear Anal. 72, 2251-2258 (2010)

2. Diblík, J, Svoboda, Z, Šmarda, Z: Retract principle for neutral functional differential equations. Nonlinear Anal., Theory Methods Appl. 71, 1393-1400 (2009)

3. Diblík, J, Růžičková, M: Existence of positive solutions of a singular initial problem for a nonlinear system of differential equations. Rocky Mt. J. Math. 3, 923-944 (2004)

4. Diblík, J, Růžičková, M, Šutá, Z: Asymptotical convergence of the solutions of a linear differential equation with delays. Adv. Differ. Equ. 2010, Article ID 749852 (2010)

5. Dorociaková, B, Najmanová, A, Olach, R: Existence of nonoscillatory solutions of first-order neutral differential equations. Abstr. Appl. Anal. 2011, Article ID 346745 (2011). doi:10.1155/2011/346745

6. Erbe, LH, Kong, QK, Zhang, BG: Oscillation Theory for Functional Differential Equations. Dekker, New York (1995)

7. Levitan, BM: Some problems of the theory of almost periodic functions I. Usp. Mat. Nauk 2(5), 133-192 (1947)

8. Lin, X: Oscillation of second-order nonlinear neutral differential equations. J. Math. Anal. Appl. 309, 442-452 (2005)

9. Lin, Z, Chen, L, Kang, SM, Cho, SY: Existence of nonoscillatory solutions for a third order nonlinear neutral delay differential equation. Abstr. Appl. Anal. 2011, Article ID 693890 (2011). doi:10.1155/2011/693890

10. Špániková, E, Šamajová, H: Asymptotic properties of solutions to $n$-dimensional neutral differential systems. Nonlinear Anal., Theory Methods Appl. 71, 2877-2885 (2009)

11. Wang, X, Liao, L: Asymptotic behaviour of solutions of neutral differential equations with positive and negative coefficients. J. Math. Anal. Appl. 279, 326-338 (2003)

12. Zhou, Y: Existence for nonoscillatory solutions of second-order nonlinear differential equations. J. Math. Anal. Appl. 331, 91-96 (2007)

13. Zhou, Y, Zhang, BG: Existence of nonoscillatory solutions of neutral differential equations with positive and negative coefficients. Appl. Math. Lett. 15, 867-874 (2002)

doi:10.1186/1687-1847-2013-140

Cite this article as: Dorociaková et al.: Uncountably many solutions of first-order neutral nonlinear differential

equations. Advances in Difference Equations 2013 2013:140.

\section{Submit your manuscript to a SpringerOpen ${ }^{\circ}$ journal and benefit from:}

- Convenient online submission

Rigorous peer review

- Immediate publication on acceptance

Open access: articles freely available online

- High visibility within the field

- Retaining the copyright to your article 